\title{
Êxito social e gênero na velhice: leitura e atividade física ${ }^{1}$ Social success and gender in elderly people: reading and physical activity
}

\author{
Geraldina Porto Witter - Universidade Camilo Castelo Branco, São Paulo, Brasil \\ Ana Paula Moreira Galvão - Nipon Country Club, São Paulo, Brasil \\ Marcelo de Almeida Buriti - Centro Federal de Educação Tecnológica de São Paulo, São Paulo, Brasil \\ Elza Maria Tavares Silva - Universidade Mogi das Cruzes, São Paulo, Brasil
}

\section{Resumo}

O objetivo da pesquisa foi estudar a ocorrência de leitura e de atividade física em idosos. Os participantes foram divididos em quatro grupos de acordo com as variáveis: gênero e êxito ou sucesso social na comunidade; cada grupo foi composto por 10 pessoas com 70 anos ou mais. Os instrumentos usados foram: Termo de Consentimento Livre e Esclarecido (TCLE), um roteiro sobre a formação do leitor e um questionário internacional sobre atividade física. Os resultados não mostraram correlação entre as duas variáveis, exceto para o grupo de homens com êxito social. Homens e mulheres com sucesso social foram melhores em leitura do que os outros grupos, mas não houve diferença estatística concernente à atividade física e gênero.

Palavras-chave: terceira idade; hábito de ler; prática de exercícios.

\section{Abstract}

The aim of the research was to study the frequency of reading and physical activity in elderly people. The participants were divided in four groups according to the following issues: gender and social success in their community; each group was composed by 10 people, at the age of 70 or more. The instruments used to collect data were: an agreement term, a schedule about the reader formation and an international questionnaire about physical activity. The results did not show correlation between both issues used to conduct the research, except in the men's group with social success. Men and women with social success showed better development in reading than the other groups, however, there was no statistical difference concerning to physical activity and gender.

Keywords: old aged; habit of reading; practice of physical activity.

\section{INTRODUÇÃO}

Dados divulgados pelas Nações Unidas (ONU) em 11 de abril de 2007, mostram que pessoas com mais de 60 anos representarão $32 \%$ da população mundial em 2050, número superior ao de crianças. Hoje a população acima dos 60 anos representa 10\% da população mundial, enquanto que em 2050 a expectativa é que esse número irá triplicar passando dos 705 milhões para quase 2 bilhões, fazendo-se necessária uma preocupação maior com os projetos e pesquisas a serem desenvolvidas para facilitar a qualidade de vida desses idosos. (http:/ / notícias.uol.com.br/ ulnot/efe/2007/04/11/ultl766u21157.jhtm).

Neste início do século XXI, a crescente globalização, as tecnologias disponíveis à população possibilitaram melhor qualidade de vida, as pessoas frequentemente planejam novas ocupações para essa etapa de vida. Nas diferentes propostas para o lazer e a ocupação do tempo livre dos idosos estão a leitura e a atividade física (AF), não se tendo, com idosos, estudos relacionando as duas áreas, embora com crianças e adolescentes essa preocupação se faça presente, e muitas vezes se use a leitura para estimular a atividade física, e vice-versa, como ocorre com outras fases da vida humana (Mason \& Schumn, 2003; Hynds, 1997). Aliás, pode-se chegar a bons resultados usando-se caminhos diferentes, como Clay (1998) demonstra bem. A leitura sobre folclore pode estimular e reviver muitas atividades físicas (Yong, 2003).

A ausência de como se relacionam leitura e atividade física em idosos recomenda que se pesquisem

\footnotetext{
${ }^{1}$ Apoio: Fundação de Amparo à Pesquisa do Estado de São Paulo (FAPESP) (Processo no 2003/11.788-6)

${ }^{2}$ Av. Pedroso de Morais, $\mathrm{n}^{\circ}$ 144, Apto 302- Pinheiros- SP - CEP: 05420-000

Tel.: (11) 3032-1967 E- mail: gwitter@uol.com.br
}

Psico-Usf, v. 14, n. 3, p. 375-386, Setembro/Dezembro 2009 
as possíveis relações entre essas variáveis na velhice, desde que em textos teóricos e em discussão de dados frequentemente é lembrada esta relação. Observou-se que na literatura há registros de pesquisas realizadas mostrando à sociedade os benefícios que são obtidos com pequenas atitudes, como o hábito da leitura na prevenção de doenças mentais, como Alzheimer, manutenção da cognição, depressão, e também a prática de atividade física no auxílio e tratamento de doenças ósseas, cardíacas, entre outras, além de proporcionarem bem estar, social e cognitivos evitando, por exemplo, solidão e depressão. Como exemplo, podem ser citados os estudos de Matsudo, T. Matsudo, V. Andrade, Andrade, Oliveira e Braggion (2002) sobre os efeitos benéficos da atividade física para o bem-estar físico e mental, caracterizando um aspecto fundamental do estilo de vida saudável do idoso.

Uma atividade relevante da velhice, e que nem todos podem desfrutar, é a leitura, dadas as condições educacionais e de vida em que sua infância e juventude transcorreram (Witter, 2006). Esta atividade tem múltiplas funções, incluindo aspectos ligados ao bem-estar (Martins, Maestri e Cosenza, 2004), e acompanha todo o ciclo de vida da pessoa e, na velhice, pode ser lazer, preenchimento do tempo, fuga de situações desagradáveis, além de um estímulo cognitivo essencial (Witter, 2005a). Mas o impacto de diversas variáveis que influem na leitura muda ao longo da vida do leitor (Rayner, Reichle, Stroud, Williams \& Pollatsek, 2006).

Para os idosos a leitura pode, como para todas as idades, assumir significados diferentes, sejam poesias, sejam histórias, livros técnicos ou notícias de jornal, a bula de um medicamento ou uma receita culinária, o seu domínio pode representar autonomia, lazer ou até mesmo um meio de se adquirir conhecimento acerca de assuntos específicos na construção do saber ou ainda sobre os benefícios da atividade física.

Segundo Jolly (1999), vários estudos mostram resultados positivos no desempenho das crianças na compreensão e leitura, comunicação e escrita, quando existe uma parceria entre a família e a escola. Os idosos podem ser personagens importantes na formação de leitores. Além disso, os que também praticam atividade física concomitantemente estão atuando nas duas áreas, sendo modelos mais completos para as novas gerações. Assim, em um mundo de constantes mudanças, o relato das histórias de leitores idosos é essencial na construção da visão da realidade e reforçam o vínculo familiar (Witter \& Passos, 2006a).

Pode-se utilizar a leitura como um recurso para o estímulo mental do idoso e também como importante instrumento, auxiliando as atividades cotidianas que ele necessita para se relacionar com o ambiente e que requerem maior participação das atividades mentais (Atividades Instrumentais da Vida Diária-AIVD) como, por exemplo, uso de equipamentos eletrônicos, celulares, movimentação bancária, uso de medicamentos, transporte coletivo etc. (Ruipérez \& Llorente, 2002). Está subjacente que a leitura pode estimular ou levar às atividades físicas diárias e mesmo estimular um maior envolvimento em grupos de ginástica e esportivos, além do que nas AIVD estão incluídas atividades de jardinagem, cuidados com a casa e exercícios relativos a alguma forma de atividade física praticada diariamente. Como exemplo cita-se a leitura de uma receita culinária (leitura) e sua execução (atividade instrumental de vida diária). Vale lembrar que pode servir de estímulo físico (Anderson, Wojcik, Winnet \& Willians, 2006).

Fatores demográficos também influenciam a AF: os níveis socioeconômico, educacional e a renda podem ser associados a níveis mais altos de AF (Barnes \& Schoenborn, 2003); quanto ao gênero, os homens praticam mais atividades vigorosas do que as mulheres, que apresentam taxas maiores de sedentarismo (Crespo, Smit, Anderson, Carter-Pokras \& Ainsworth, 2000), ressaltando-se que a prática de AF moderada é considerada suficiente para se alcançar efeitos benéficos para saúde (Anderson, Wojcik, Winett \& Willians, 2006). Barnes e Schoenborn (2003) lembram que apesar da AF moderada declinar com a idade, alguns dados sugerem que a $\mathrm{AF}$ vigorosa entre pessoas de 65 a 74 anos de idade é mais comum do que entre jovens e adultos de meia-idade (Brownson, Jones, Pratt, Blanton \& Heath, 2000). Assim, é viável o objetivo de se introduzir a prática da AF na rotina dos idosos, e isso poderá refletir em sua qualidade de vida, que, segundo Forti e Rolim (2004), relaciona-se com o fato de se sentirem melhores, conseguirem cumprir com suas funções diárias básicas adequadamente e estarem aptos a viver de forma independente. Segundo Donaldson (2000), essa prática deveria ser considerada uma das principais intervenções para a melhoria da saúde pública, atuando como fator de prevenção de doenças crônicas, promoção da saúde, menor risco de quedas e aumento da qualidade de vida dos idosos, inclusive entre os que se encontram em asilos (Galvão \& Buriti, 2006).

Em relação ao praticar atividades físicas, diferentes pesquisas usaram o instrumento Questionário Internacional de Atividade Física (IPAQ), contudo, os resultados mostram que há diferença na incidência da classificação "sedentário", enquanto o grupo estudado no ambiente asilar teve grande número de sedentários (Galvão \& Buriti, 2006), o grupo de brasileiros teve um número razoável (Matsudo \& cols, 2002) e o 
grupo de descendentes japoneses não teve nenhum participante com a mesma classificação (Buriti, Talarico, Galvão, Silva \& Buriti, 2006).

Embora seja frequente a menção da relevância tanto da leitura como da atividade física para o idoso, pouco se fez de pesquisa desde o clássico livro de Gentili (1980) que estabelece uma relação positiva entre esporte, educação física e fortalecimento das habilidades de leitura decorrentes da necessidade das pessoas conhecerem mais sobre AF. Sugere, com base em vivências e em alguns dados, a produtividade da relação. Entretanto, pouco se tem de dados de pesquisa, especialmente com idosos.

São raros os trabalhos como o de Sallis e Hovell (1990), em que se encontram informações que permitem relacionar leitura com prática de AF, mas ainda sem estar especificamente enfocando o idoso.

Pesquisas de metaciência sobre a velhice apontam muitas lacunas nas pesquisas realizadas, quer quanto aos tipos de trabalho, quer quanto aos temas enfocados (Witter \& Assis-Maria, 2005; Witter \& Ferreira, 2005). Entre as ausências notadas está a busca de possíveis relações entre leitura e AF. Embora na prática ocorram exemplos diversos, ainda há carência de dados.

Pelo exposto, leitura e AF podem contribuir para a qualidade de vida e a saúde de aspectos afetivos, cognitivos, sociais e físicos, todavia a relação entre ambas é praticamente desconhecida no idoso, podendo ser trabalhadas concomitantemente em programas para idosos. Entretanto, inicialmente, é necessário verificar como estão ocorrendo na realidade, para se ter dados que permitam futuramente planejar intervenções. Em atividade com grupos de idosos é possível, e uma das autoras já usou o recurso várias vezes - mas sem o contexto de pesquisa de recorrer à leitura para estimular idosos sedentários a buscar atividades físicas, que se tornaram prazerosas e constantes em suas práticas, bem como manter a aderência a tratamentos por vezes dolorosos, mas essenciais.

Tomando por base as contingências diversificadas de vida que vivenciam as atuais gerações de idosos, as sugestões da literatura sobre uma possível relação entre leitura e atividade física e a possibilidade já vivenciada na prática de usar uma para estimular a outra, sendo ambas relevantes para a vida dos idosos, considerou-se necessário conhecer como ponto de partida a vivência deles com as duas atividades e, posteriormente, detectar uma possível relação entre elas. Assim sendo, foram estabelecidos como objetivos específicos: (1) verificar a leitura em idosos, tendo em vista gênero e destaque social; (2) analisar a atividade física dos idosos com e sem destaque social, bem como quanto à gênero; para, em seguida, (3) verificar se há correlação entre leitura e AF nos referidos grupos.

\section{MÉTODO \\ Participantes}

Foram constituídos quatro grupos de participantes amostrados por conveniência do banco de dados de pesquisa sobre idosos em cidade do interior paulista. Os critérios comuns para inclusão no grupo foram ter no mínimo 70 anos e residir na cidade há pelo menos 20 anos. Desta forma foram selecionados 40 idosos, sendo metade de cada gênero. Em cada gênero foram compostos dois grupos: um constituído por pessoas comuns e outro por pessoas com algum destaque social, isto é, influentes na sociedade em decorrência de alguma variável (artistas, industriais, atletas de alto desempenho, posição financeira, políticos, sindicalistas etc.), independentemente de escolarização e poder econômico. Optou-se pelo uso da presente classificação, que está se tornando progressivamente mais usada e permite considerar a pessoa como é vista pela sociedade, pelos que com ela convivem, independentemente do conceito de classe social, que já não atende plenamente às contingências biopsicossociais da pós-modernidade e da linguagem politicamente correta em pesquisa. As de destaque social são pessoas cujos comportamentos são mais provavelmente tomados como modelos, como exemplo, e criticadas quando não correspondem ao que delas se espera. Estão frequentemente nos jornais, integrando e liderando movimentos sociais, participando de solenidades públicas e de outras atividades na sociedade. São reconhecidas pela maioria das pessoas da comunidade como pessoas que, de alguma for$\mathrm{ma}$, se destacaram. A partir dessas características, buscou-se, em uma cidade do interior, idosos para completar os grupos de pesquisa de um grande projeto (FAPESP - Proc. N N $^{\circ}$ 2003/11.788-6). Os sujeitos foram encontrados em situações de atividades destinadas a idosos na cidade ou em suas residências. Deste grupo maior de idosos foram sorteados os 40 participantes do presente estudo. Os quatro grupos ficaram assim constituídos:

- GFA composto por 10 mulheres de destaque na cidade, tendo idade entre 70 e 80 anos, com média de 77 anos. O tempo de vivência na cidade variou de 42 a 80 anos, sendo de 65 anos a média.

- GFB composto por 10 mulheres sem destaque na cidade, tendo idade entre 70 e 90 anos, com média de 75 anos. Habitavam na cidade em média há 43 anos, em uma variação de 30 até 67 anos.

- GMA composto por 10 homens de destaque na cidade, tendo idade variando entre 70 e 83 anos, sendo a média de 77 anos. Residiam na cidade de 46 a 83 anos, com média de 70 anos. 
- GMB o grupo de 10 homens comuns tinha idade entre 73 e 86 anos, com média de 79 anos. Moravam na cidade entre 40 a 83 anos, com média de 60 anos.

\section{Material}

Os materiais utilizados para efetivar a coleta foram:

Termo de Consentimento Livre e Esclarecido (TCLE) aprovado com o projeto pelo Comitê de Ética em Pesquisa (CEP 64/ 2003), pelo qual o participante lavrava sua anuência em ser sujeito do estudo e era informado sobre objetivos, método do trabalho, em que consistia sua participação e sobre seus direitos.

Formação de leitores: a partir de um roteiro de entrevista elaborado por Witter e Oliveira (1997) e também usado por Ruiz (2003), a que se incluíram alguns itens, foi estruturado o instrumento, o qual foi usado anteriormente por Witter e Passos (2006 a; 2006 b), Witter e Queiroz (2006). Consiste em solicitar do participante relatar desde a sua primeira lembrança envolvendo a leitura até como ela ocorre na sua vida atual. Para atribuição de um valor síntese foi instituído um índice de leitura mediante o critério de avaliação de cada protocolo, conforme aparece na Tabela 1. A pontuação foi estabelecida em relação ao valor demonstrado na literatura pelas variáveis a que os participantes referiram em sua trajetória como leitores. Viabilizou-se com estes critérios estabelecer um total de pontos para cada idoso para permitir a quantificação e análise estatística. A pontuação final é um índice do idoso enquanto leitor (formação e atuação). 
Tabela 1- Critério de Pontuação da Leitura

\begin{tabular}{lcll}
\hline 1) Niveis de escolaridade & Pontos & 2) Lembranças de leitura & \\
Ensino (primário completo) & 1 & na Infância & Pontos \\
Médio (completo) & 2 & Sem lembrança & 0 \\
Superior Incompleto & 3 & Lembrança de livro & 1 \\
Superior Completo & 4 & Lembrança de atividade & 1 \\
Lato Sensu & 4 & & \\
Stricto Sensu (Mestrado) & 4 & & \\
Stricto Sensu (Doutorado) & 5 &
\end{tabular}

3) Lembranças de Leitura

na Adolescência

Pontos

4) Lembranças de aspectos da trajetória

Sem lembrança

0

como leitor

Pontos

Lembrança genérica

1

Lembrança específica

1

Lembrança genérica

1

Lembrança de pessoas

1

Lembrança de método

1

Lembrança de idade ? 7 anos 1

Lembrança $>7$ anos

0

5) Lembrar de áreas e apreciação Pontos

6) Motivação Infância/Adolescência

Sem Lembrança

Lembrança de áreas do conhecimento

1

Lembrança com apreciações positivas

1

$-1$

Lembrança com apreciações negativas

7) Pessoas influentes

Pontos

8) Motivação/adulto

Pontos

Pai/Mãe/Professores

2 (p/cada)

Gosta Muito

Pontos

Avós e outros parentes

Colega

1

Gosta

2

1

Não Gosta

2

Autores

Pontos

10) Impacto na leitura dos filhos

Pontos

Sim

2

Sim

2

Não

0

Não

0

11) Lê para e com os netos

Sim

Não
Pontos

2

0
12) Impacto na leitura dos netos

Pontos

Sim

2

Não 
Questionário Internacional de Atividade Física (IPAQ): é instrumento validado em uma amostra brasileira por Matsudo e colaboradores (2001), usado por outros pesquisadores como, por exemplo, Galvão e Buriti (2006).

O IPAQ versão curta contém perguntas em relação à frequência e duração da $\mathrm{AF}$ moderada, vigorosa e de caminhada, realizadas pelo avaliado durante a última semana. Considerando-se as atividades realizadas no trabalho, em casa, no jardim, como lazer ou exercício e que produzem gasto calórico. Para viabilizar comparar com outro instrumento, foi estabelecido um índice de atividade física que consiste na soma de pontos obtidos pelos participantes. A pontuação de AF seguiu o seguinte critério de pontuação: atividade leve para cada dia da semana 1 ponto, para cada hora da semana 1 ponto; atividade moderada para cada dia da semana 1 ponto, para cada hora ponto e atividade vigorosa 1 ponto por dia da semana e 2 pontos por hora de atividade. A soma de pontos é um índice de atividade física desenvolvida pela pessoa.

\section{Procedimento}

Após aprovação pelo Comitê de Ética em Pesquisa com Seres Humanos foi feito o contato com os idosos, explicada a pesquisa, lido o TCLE e assinado pelos que concordaram em participar (Proc. $n^{\circ} 2003 / 11.788-6$ ). Em seguida foi aplicado o roteiro sobre leitura e, após isto, o IPAQ. Os sujeitos já participavam do grupo pesquisado pelos autores. Em algumas situações foram solicitados a colaborar quando estavam fazendo alguma atividade para idosos. Então, ao concordarem, eram atendidos individualmente e feita a aplicação na ordem prevista. Para alguns participantes, principalmente os de destaque, todos ainda muito ativos na sociedade ou na vida profissional, foi marcada hora em suas casas ou locais de trabalho. No mais, foi seguido o mesmo procedimento.

\section{RESULTADOS E DISCUSSÃO}

A análise dos dados foi feita recorrendo-se aos procedimentos da estatística não-paramétrica considerada adequada ao número de participantes em cada subgrupo, ao tipo de instrumento usado e ao seu nível de mensuração. Estabeleceu-se como margem de erro aceitável $\mathrm{p} \leq 0,05$.

Para as comparações entre os grupos, levando em consideração concomitantemente as variáveis gênero e condição social, foi usado o procedimento ANOVA não-paramétrica em face da características da amostra e dos instrumentos utilizados (Wilcox \& Wilcoxon, 1964). Manteve-se a margem de erro aceita para a pesquisa. Definiu-se como $\mathrm{H}_{\mathrm{o}}$ que todos os grupos apresentavam o mesmo desempenho e por $\mathrm{H}_{\mathrm{a}}$ que seriam diferentes, mas sem especificar a direção das diferenças (Wilcox e Wilcoxon, 1964), sendo $\mathrm{k}=4$ e $\mathrm{n}=10, \mathrm{~d}_{\mathrm{c}}=134,3$.

Inicialmente são apresentados os dados relativos a cada variável, e em seguida é feita a correlação entre elas, seguindo-se a ordem dos objetivos.

Tabela 2- Comparação entre os grupos no índice de leitura.

$\mathrm{d}_{\mathrm{c}}=134,3$ e pd" 0,05

\begin{tabular}{|c|c|c|c|c|}
\hline Grupos & $\begin{array}{l}\text { GFB } \\
305\end{array}$ & $\begin{array}{c}\text { GMB } \\
287\end{array}$ & $\begin{array}{l}\text { GFA } \\
135,5\end{array}$ & $\begin{array}{c}\text { GMA } \\
92,5\end{array}$ \\
\hline$\overline{\text { GFB }}$ & & & & \\
\hline $\begin{array}{l}305 \\
\text { GMB }\end{array}$ & - & - & - & - \\
\hline $\begin{array}{l}287 \\
\text { GFA }\end{array}$ & 18 & - & - & - \\
\hline $\begin{array}{l}135,5 \\
\text { GMA }\end{array}$ & $169,5^{*}$ & $151,5^{*}$ & - & - \\
\hline 92,5 & $212,5^{*}$ & $190,0^{*}$ & 43 & - \\
\hline
\end{tabular}

Aplicando-se ANOVA na variável leitura, os grupos apresentaram diferença significante entre o grupo feminino "B" e "A" $(169,5)$, o grupo masculino "B" e o feminino "A" $(151,5)$, o grupo feminino "B" e grupo masculino " $\mathrm{A}$ " $(212,5)$ e o grupo masculino "B" e "A" (190) para d $=134,3$ e $\mathrm{p} \leq 0,05$, resultados que permitem a rejeição de $\mathrm{H}_{\mathrm{o}}$ nestes casos. Como o menor número de pontos indica melhor desempenho, foi o GMA que mais se envolveu com leitura, seguido pelo GFA, GMB e GFB (Tabela 2).

Em face das diferenças detectadas pela ANOVA e tendo em vista os objetivos da pesquisa, recorreuse ao teste U para as comparações necessárias, tendo por $\mathrm{H}_{\mathrm{o}}$ que eram iguais e por $\mathrm{H}_{a}$ que eram diferentes. 
Manteve-se $\mathrm{p} \leq 0,05$. No caso de leitura a comparação entre os gêneros, resultou em $\mathrm{R}_{1=} 379,5$ para os homens e $R_{2}=440,5$ para as mulheres, sendo $U_{o=} 169,5$, como $\mathrm{N}_{1}=20$ e $\mathrm{N}_{2}=20, \mathrm{U}_{\mathrm{c}}=127$, podendo-se concluir que a hipótese nula não foi rejeitada, tendo o grupo masculino melhor desempenho, mas sem significância estatística. Os homens foram apenas pouco mais escolarizados do que as mulheres $(67,7 \%$ contra $62,6 \%$, respectivamente), já que até os anos 60 tiveram mais acesso à escola do que as mulheres, dados segundo o censo de 2000 (IBGE). Outro dado do IBGE é em relação à média de anos de estudo, que nas capitais era e é bastante superior à zona rural, sendo que alguns participantes da presente pesquisa se originaram da região rural, o que pode explicar em parte o resultado, pois muitos só na idade adulta vieram para a zona urbana, para uanto ao destaque social, o GA obteve-se $R_{1=} 228$ e para GB R $2=379,5$, neste caso, mantidas as condições usadas para gênero, $\mathrm{U}_{\mathrm{o}=1} 18$, inferior ao valor crítico do $\mathrm{p}$ adotado. Podese concluir que as pessoas com destaque social apresentaram melhor desempenho estatístico em leitura, o que era esperado, tendo em vista a contarem também com mais anos de escolarização.

Witter \& Passos (2006b) realizaram pesquisa sobre a formação de leitores descendentes japoneses com idade superior a 70 anos, com ou sem destaque social. Em seus resultados observaram que em ambos os grupos a figura do professor foi a mais mencionada como o motivador da leitura em suas vidas, seguida pelo pai, mãe e autores, ao responderem sobre a sua lembrança de leitura fora da escola, $60 \%$ dos integrantes do grupo com destaque afirmaram tê-la, enquanto $50 \%$ do grupo sem destaque afirmou o contrário. As mesmas autoras (2006a) quando comparam a relação entre leitura e a presença ou não de destaque social em um grupo brasileiro, observaram que apesar de ambos os grupos valorizarem a leitura, apenas o GA (com destaque) possui as lembranças relacionadas à leitura fora do ambiente escolar e que o GB relatou dificuldade na aquisição de livros e no uso de bibliotecas públicas na infância e juventude.

As pesquisadoras constataram nos relatos colhidos nas diferentes amostras étnicas que os grupos com destaque social, por terem nível socioeconômico e cultural mais bem situados que os grupos sem destaque, têm melhores condições para a aquisição e manutenção dos hábitos de leitura e ambiente mais favorável para o desenvolvimento da leiturabilidade em família. Já os grupos sem destaque relataram dificuldades financeiras para a aquisição de livros e falta de tempo livre em virtude da ocupação com obrigações, até mesmo quando eram crianças. O mesmo é válido para os dados aqui relatados e aplica-se também para AF planejada. Dados similares aparecem em Tessaro (2003) e Martins, Maestri e Cosenza (2004).

$\mathrm{Na}$ Tabela 3 estão dispostos os dados da ANOVA para a comparação entre todos os grupos e variáveis, quanto ao índice de atividade física. O GFB alcançou o primeiro lugar, seguido pelo GMB, GFA, GMA.

Tabela 3- Comparação entre os grupos no índice de atividade física.

$\mathrm{d}_{\mathrm{c}}=134,3$ e pd" 0

\begin{tabular}{|c|c|c|c|c|}
\hline Grupos & $\begin{array}{c}\text { GMA } \\
245\end{array}$ & $\begin{array}{c}\text { GFA } \\
220 \\
\end{array}$ & $\begin{array}{c}\text { GMB } \\
189\end{array}$ & $\begin{array}{c}\text { GFB } \\
166\end{array}$ \\
\hline GFB & & & & \\
\hline $\begin{array}{l}245 \\
\text { GMB }\end{array}$ & - & - & - & - \\
\hline $\begin{array}{l}220 \\
\text { GFA }\end{array}$ & 25 & - & - & - \\
\hline $\begin{array}{l}189 \\
\text { GMA }\end{array}$ & 56 & 31 & - & - \\
\hline 166 & 79 & 54 & 23 & - \\
\hline
\end{tabular}

Os resultados, apesar de demonstrarem que o grupo GFB tem melhor desempenho diante dos demais grupos, teve diferenças não estatisticamente significantes. Utilizando-se o mesmo critério adotado para leitura, nenhum dos grupos superou os valores de $\mathrm{d}_{\mathrm{c}}=134,3$ na avaliação de atividade física e $\mathrm{H}_{\mathrm{o}}$ não foi rejeitada.

Ao utilizar o teste $\mathrm{U}$ para as outras comparações intergrupos, manteve-se $\mathrm{p} \leq 0,05$ e por $\mathrm{H}_{\mathrm{o}}$ que os grupos eram iguais e por $\mathrm{H}_{2}$ que eram diferentes. 
Quanto ao destaque social no $\mathrm{GA}$, obteve-se $\mathrm{R}_{1=} 465$ e no $G B R_{2=} 355$, neste caso, mantidas as condições usadas para leitura, $\mathrm{U}_{\mathrm{o}=} 145$ e $\mathrm{U}_{\mathrm{c}}=127$, portanto, superior ao valor crítico do $p$ adotado, novamente a $\mathrm{H}_{\mathrm{o}}$ não foi rejeitada. Pode-se concluir que o grupo sem destaque social apresentou melhor desempenho em atividade física, mas não foi suficiente para se afirmar que haja superioridade estatística em relação ao outro grupo.

Possivelmente pelo fato do GB ser um grupo que muitas vezes mantém uma rotina diária de afazeres e o instrumento utilizado (IPAQ) adotar como atividade física além das atividades planejadas, como por exemplo, ginástica, dança, natação, também os afazeres domésticos, os deslocamentos entre locais que consomem acima de dez minutos de caminhada, como ir à padaria, supermercado ou buscar os netos na escola, o resultado tenha sido favorável aos seus integrantes, equilibrando a AF nos grupos. É um aspecto a merecer mais pesquisas. Cumpre lembrar a necessidade de mais pesquisas das características cientométricas do instrumento e das relações entre suas partes.

Quando foi comparado o gênero no caso de AF, o masculino obteve $R_{1=} 434$ e o feminino $R_{2=} 386$; sendo $\mathrm{U}_{\mathrm{o}=1} 176$ e $\mathrm{U}_{\mathrm{c}=} 127$, conclui-se que a hipótese nula não foi rejeitada, tendo o grupo feminino melhor desempenho em AF, mas o valor alcançado não refletiu significância estatística.

Crespo, Smit, Anderson, Carter-Pokras e Ainsworth (2000) observaram diferença quanto à prática de atividade física vigorosa entre os gêneros, os homens praticando mais atividades vigorosas do que as mulheres e estas, por sua vez, apresentando taxas maiores de sedentarismo. Tais resultados não se confirmaram no presente estudo com idosos. Também os resultados de Sallis e Hovell (1990) obtidos estão em concordância com os da pesquisa mencionada anteriormente na comparação entre os gêneros, sendo os homens mais ativos. Entretanto, a diferença pode não ser estatisticamente significante, pois não foi calculada pelos autores, resultado similar ao de Matsudo e colaboradores (2002). Mas aqui também os dados são expressos apenas em percentuais sem cálculo de significância. Como para tanto é necessário que as diferenças, os dados novamente não permitem estatisticamente afirmar a superioridade de um grupo sobre o outro, ou seja, coincide com os resultados do presente trabalho. O mesmo ocorreu com os dados de Galvão e Buriti (2006), em que as diferenças também estão aquém da significância estatística. Já, Pereira e colaboradores (1999) constataram que o gênero feminino era mais ativo que o masculino na pesquisa realizada em Novo Hamburgo/RS., onde a diferença foi de apenas $2 \%$, sem significância estatística. Na pesquisa realizada por Rosa, Geraldo e Bueno de Avilla (2005), novamente se observou a superioridade feminina na classificação ativo, que foi de $10 \%$ em relação aos homens, mas a diferença foi insuficiente para ficar na região de rejeição de $\mathrm{H}_{\mathrm{o}}$

Conhecida a situação relativa às variáveis, foi estabelecida a correlação de postos (Siegel, 1956) entre leitura e atividade física em cada grupo, para os subtotais por condição social e gênero e para o total geral dos participantes (Tabela 4).

Tabela 4 - Correlação entre leitura e atividade física por destaque social e gênero.

\begin{tabular}{ll}
\hline Grupos & $\mathrm{r}_{\mathrm{o}}$ \\
\hline GFB & $-0,22$ \\
GFA & $-0,35$ \\
GMA & $0,80 *$ \\
GMB & $-0,10$ \\
GM & $-0,09$ \\
GF & $-0,05$ \\
GA & 0,24 \\
GB & $-0,08$ \\
Total & $-0,13$ \\
\hline
\end{tabular}

$*$ Significante na margem de erro aceita para a pesquisa $\left(\mathrm{r}_{\mathrm{c}}=0,58\right)$. 
Só houve correlação positiva entre leitura e AF no grupo masculino com destaque social, $\mathrm{r}_{\mathrm{o}}=0,80 \mathrm{e}$ $r_{c=} 0,58$, porém quando este foi unido com a mesma categoria, só que feminina, essa correlação desapareceu, significando que não houve significância no grupo como um todo. $\mathrm{O}$ resultado demonstrou que o grupo masculino com destaque social lê e pratica $\mathrm{AF}$ de forma hierarquicamente similar.

Pode-se lembrar Andreotti e Okuma (2003) que em pesquisa para estabelecer os fatores sociodemográficos que influenciam a adesão em um programa de AF por idosos, verificaram que o gênero, idade, grau de instrução, renda (status socioeconômico), raça e etnia, bem como estado civil, podem interferir positiva ou negativamente. Parece que no presente caso o grau de instrução que implica condição de leitura está variável a merecer mais estudos, especialmente no, que concerne a suas relações com os vários tipos de atividade física já que é um aspecto carente de evidências, mas referido como relevante na literatura.

Os dados aqui relatados não corroboram a diferença de gênero em AF. Já Barnes e Schoenborn (2003) encontraram relação positiva entre maior nível educacional e AF, o que não tendeu a acontecer entre os idosos aqui pesquisados, pois os de destaque social, de ambos gêneros, ou seja, os leitores de maior pontuação apresentaram piores resultados em atividade física do que o obtido pelos participantes do GB.

\section{CONSIDERAÇÕES FINAIS}

É conveniente lembrar a carência de instrumentos cientificamente sólidos na área. Recorreu-se a um instrumento de amplo uso internacional, mas que carece de pesquisas de validação, precisão, fidedignidade e não conta com parâmetros para comparação. $\mathrm{O}$ outro instrumento é um roteiro de entrevistas e, para efeito de viabilizar a comparação pretendida, foi pontuado tendo por base a literatura sobre a matéria. Em ambos os casos, é preciso muita cutela na interpretação e generalização dos resultados.

Em relação à leitura, o destaque social favoreceu as condições de leitura dos participantes, assim, fazer parte de um grupo socioeconômico e cultural mais evidente parece ter influenciado positivamente os idosos, mas o mesmo não ocorreu quanto à variável gênero, que não apresentou, diferenças com significância estatística. São aspectos a merecer novos estudos.

Fazer parte de um grupo com destaque social não influenciou na prática de exercícios físicos, o que também foi verificado quanto ao gênero. Apesar de o grupo sem destaque social e o gênero feminino terem apresentado melhor desempenho, a análise estatística não indicou que estes são superiores ao grupo com destaque social e ao gênero masculino.

A correlação entre leitura e atividade física foi estabelecida apenas no grupo masculino com destaque social, contudo não foi possível afirmar que ela existiu no grupo bem-sucedido como um todo, de forma a se manter a mesma hierarquia de desempenho entre estes participantes nas duas variáveis. Nos demais grupos não houve correlação, indício de que os sujeitos convivem em universos diferentes, requerendo necessidades específicas para sua prática.

Os dados indicam que há mais homogeneidade entre os grupos no que concerne à prática de atividade física do que em leitura. Possivelmente a escolaridade, as necessidades de atuação e a maior presença em atividades físicas da comunidade seja uma variável subjacente ao encontrado. Também pode ter sido uma variável a influir nos dados a ênfase que a necessidade de AF como meio para saúde tem nos meios de comunicação de massa, nos consultórios e mesmo na família. Isso não ocorre com a leitura. Todavia, há muito por pesquisar na área, e como as várias gerações de idosos viveram contingências sócio-históricas e culturais distintas há que se considerar esses contextos na determinação de suas opções de vida, inclusive em leitura e AF.

Certamente, outras pesquisas se fazem necessárias para se conhecer mais a problemática. Possivelmente, autorregistros de AF e de leitura possam ser mais eficientes e seguros para um conhecimento mais específico das variáveis em tela ou mesmo a observação, ou ainda outros instrumentos indiretos de avaliação.

\section{REFERÊNCIAS}

Anderson, E. S.; Wojcik, J. R., Winett, R. A. \& Willians, D. M. (2006). Social-Cognitive Determinants of Physical Activity: The Influence of Social Support, Self-Efficacy, Outcome Expectations, and Self-Regulation Among Participants in a Church-Based Health Promotion Study. Health Pychology. 4:510-520.

Andreotti, M. C. \& Okuma, S. S. (2003). Perfil sóciodemográfico e de adesão inicial de idosos ingressantes em um programa de Educação Física. Revista Paulista de Educação Física. 17 (2). 142: 153.

Barnes, P. M. \& Schoenbon, C. A. (2003). Physical Activity among adults. Advanced Data. 333:1-23.

Buriti, M. S. L., Talarico, L. M., Galvão, A. P. M., Silva, M. A. da \& Buriti, M. de A. (2006) in G. P. Witter. Envelhecimento: referenciais teóricos e pesquisas. Cap 8, p. 155-176 
Brownson, R. C.; Jones, D. A. ; Pratt, M., Blanton, C. \& Heath, G. W. (2000). Measuring physical activity with the behavioral risk factor surveillance system. Medicine and Science in Sports and Exercise. 321:1913-1918.

Clay, M. M. (1998). By different paths to common outcomes. York, Maine: Stenhouse Publisher.

Crespo, C. J.; Smit, E.; Anderson, R. E., Carter-Pokras, O. \& Ainsworth, B. E. (2000). Race/Ethnicity, social class, and their relation to physical Activity during leisure time: Results from the Third National Health and Nutrition Examination Survey, 1988-1994. American Journal of Preventive Medicine. 18:46-53.

Forti, V. A. M. \& Rolim, F. S. (2004). Envelhecimento e atividade física: auxiliando na melhoria e Manutenção da qualidade de vida. In: Diogo, M. J. D. ; Néri, A. L. e Cachione, M. ( Org). Saúde e qualidade de vida na velhice. Campinas: Alínea.

Galvão, A. P. M \& Buriti, M. de A. (2006). Nível de atividade Física em idosos instucionalizados. $6^{\circ}$ Congresso Nacional de Iniciação Cientifica-CONIC e $4^{\circ}$ Congresso Internacional de Iniciação Cientifica-COINT. Guarulhos/SP.

Gentili, L. M. (1980). Using sports and physical education to strengthen reading skills. Newark: IRA, 1980.

Hynds, S. (1997). On the brink: negotiating literature and life with adolescents. Newark, Del: IRA.

Joly, M. C. R. A. (1999). Leitura: o que sabemos o que precisamos saber (influência da família na alfabetização) In: Witter, G. P. Leitura textos e pesquisas. Campinas. Alínea.

Martins, L. N. R., Maestri, M. \& Cosenza, M. (2004). Contexto de leitura em professores universitários. In: G. P. Witter. Leitura e Psicologia. Campinas. Alínea.

Mason, P. A. \& Schumn, J. S. (2003). Promissing practices for urban reading instruction. Newark, Del: IRA.

Matsudo, S. Araújo, T. Matsudo, V. Andrade, D., Andrade, E, Oliveira, L. \& Braggion, G. (2001). Questionário Internacional de Atividade Física (IPAQ): estudo de validade e reprodutibilidade no Brasil. Revista Atividade Física \& Saúde, 6(2)5-18.

Matsudo, S. Araújo, T. Matsudo, V. Andrade, D., Andrade, E., Oliveira, L. \& Braggion, G. (2002). Nível de atividade física da população do Estado de São Paulo: análise de acordo com o gênero, idade nível socio-econômico, distribuição geográfica e de conhecimento. Revista Brasileira Ciência e Movimento, 10(4): 41-50.
Pereira, F.P.; Reichert, C. L.; Costa, T. K.; Dalsasso, L. \& Lopes, L. (1999). Análise quantitativa de indivíduos adultos que praticam atividade física regular norma (ACSM, 1998). Em Novo Hamburgo - RS. In Anais XXII Simpósio Internacional de Ciência do Esporte: Atividade Física: Da Comunidade ao Alto rendimento. p7.

Rayner, K.,; Reiche, E. D.; Stroud, M. J., Willians, C. C. \& Pollatsek, A. (2006). The effect of word frequency, word predictability, and font difficulty on the eye movements of young and olders readers. Psychology and Aging. 21 (3), 448-465.

Rosa, F. G.; Geraldo, M. \& Bueno de Avilla (2005). Qualidade de vida, Atividade física e Envelhecimento. A Terceira Idade. 16 (32)52: 65.

Ruipérez, I. \& Llorente, P. (2002). Geriatria: guias práticos de enfermagem. Rio de Janeiro. Mc Graw Hill.

Ruiz, V. M., (2003). Autobiografia como leitor - um estudo com primeiristas de cursos noturnos de letras e pedagogia. Leitura e Psicologia. Campinas. Alínea.

Sallis, J. F. \& Hovell, M. F. (1990). Determinants of exercise behavior. Exercise and Sciences Review.18. 307:330.

Siegel, S. (1956). Nonparametric Statistics: for the behavioral sciences. New York: Mc Graw-Hill Book Co.

Tessaro, N. S. (2003). Leitura na vida de universitários: Estudo comparativo entre instituições de ensino superior. In: Witter, G. P. (Org). Leitura e Psicologia. Campinas: Alínea.

Wilcoxon, W. \& Wilcox, R. A. (1964). Some Rapid approximates statistical procedures. New York: Lederle Laboratories.

Witter, G. P. (org) (2005b). Metaciência e Psicologia. Campinas, SP: Editora Alínea.

Witter, G. P. (2006). Leitura e Idoso. In G. P. Witter (org.). Envelhecimento referenciais teóricos e pesquisas. Campinas: Alínea.

Witter, G. P. \& Oliveira, M. H. M., (1997). História de leitores: Pós graduandos em Psicologia Escolar. In: Witter. G. P. (Org). Psicologia: leitura e universidade. Campinas: Alínea.

Witter, G. P. (2005a). Leitura no Reading Research Quarterly (2000/2002). In G. P. Witter (org.): Metaciência e Psicologia. Cap. 7, 137-154. Campinas, SP: Alínea.

Witter, G. P. \& Ferreira, A. A. (2005). Idoso e leitura: análise da produção científica arrolada na PsycINFO (2001/2003). In G. P. Witter (org.): Metaciência e Psicologia. Cap. 8, 155-170. Campinas, SP: Alínea. 
Witter, G. P. \& Assis-Maria, F. (2005). Velhice no Banco de Teses da CAPES (2000/2001). In G. P. Witter (org.): Metaciência e Psicologia. Cap. 9, 171-184. Campinas, SP: Alínea.

Witter, G. P. \& Passos, S. P., (2006a). Formação de leitores: relatos de leitores com ou sem destaque social. In: Witter, G. P. (org). Envelhecimento: referenciais teóricos e pesquisas. Campinas: Alínea.

Witter, G. P. \& Passos, S. P., (2006b). Formação de leitores de origem japonesa. In: Witter, G. P. (org). Envelhecimento: referenciais teóricos e pesquisas. Campinas: Alínea.
Witter, G. P. \& Queiroz, N. B. (2006). In G. P. Witter. Envelhecimento: referenciais teóricos e pesquisas. Cap. 13, p. 271-284.

Yong, T. A. (2003). Happily ever after: sharing folk literature with elementary and middle school students. Newark, Del: IRA.

\section{Sobre os autores:}

Geraldina Porto Witter - UNICASTELO

Ana Paula Moreira Galvão - NCC Nipon Country Club

Marcelo de Almeida Buriti - USJT/CEFET/UMC 